PROCEEDINGS OF THE

AMERICAN MATHEMATICAL SOCIETY

Volume 140, Number 5, May 2012, Pages 1823-1834

S 0002-9939(2011)11007-4

Article electronically published on August 24, 2011

\title{
LOW DEGREE REPRESENTATIONS OF SIMPLE LIE GROUPS
}

\author{
ROBERT GURALNICK, MICHAEL LARSEN, AND COREY MANACK \\ (Communicated by Gail R. Letzter)
}

\begin{abstract}
We show that the number of irreducible representations of degree $\leq n$ of any simple compact Lie group $G$ is $\leq n$. If the rank of $G$ is large, the bound is much smaller. A consequence is that the number of conjugacy classes of closed maximal subgroups for a simple compact or complex Lie group of rank $r$ is $O(r)$.
\end{abstract}

\section{INTRODUCTION}

We say that a compact Lie group is simple if it is connected, has finite center and is a simple group modulo its center. This paper is mostly concerned with upper bounds for $R_{n}(G)$, the number of characters of $G$ of degree $\leq n$. It is convenient to define the Witten zeta-function

$$
\zeta_{G}(s)=\sum_{\chi} \chi(1)^{-s}
$$

where the sum is taken over all irreducible characters of $G$. By [LL, the abscissa of convergence of this Dirichlet series is $2 / h$, where $h$ denotes the Coxeter number of $G$. This implies that

$$
R_{n}(G)=O\left(n^{2 / h+\epsilon}\right)
$$

for all $\epsilon>0$. One can turn this around and try to bound $R_{n}(G)$ for fixed $n$ and varying $G$. In this direction, Wallach [W2] proved that $R_{n}(G) \leq 7 n$.

Our first result gives the optimal bound for this problem.

Theorem 1. If $G$ is a simple compact Lie group and $n$ is a positive integer,

$$
R_{n}(G) \leq n
$$

with equality only if $n=1, G=\mathrm{SU}(2)$, or $G=\mathrm{SU}(3)$ and $n=3$.

If we exclude groups of low Coxeter number, we can improve on Theorem 1. A formulation in terms of the Witten zeta-function is as follows.

Theorem 2. If $G_{1}, G_{2}, \ldots$ is a sequence of pairwise non-isomorphic simple compact Lie groups, and $s>0$, then

$$
\lim _{n \rightarrow \infty} \zeta_{G_{n}}(s)=1 .
$$

Received by the editors March 22, 2010 and, in revised form, December 9, 2010; January 4, 2011; and January 13, 2011.

2010 Mathematics Subject Classification. Primary 22C05, 22E46.

The authors were partially supported by NSF Grants DMS-0653873, DMS-1001962 and DMS0800705 .

(C)2011 American Mathematical Society Reverts to public domain 28 years from publication 
This can be regarded as an analogue for compact Lie groups of the main theorem of [LS]. An immediate consequence of Theorem 2 is

Corollary 3. For every $\epsilon>0$ there exists $N$ such that if $G$ is a simple compact Lie group of dimension $\geq N$, then $R_{n}(G) \leq n^{\epsilon}$ for all $n \geq 1$.

As a further application, we prove the following theorem.

Theorem 4. For all $\epsilon>0$, the number of isomorphism classes of pairs $(G, V)$, where $G$ is a compact semisimple group and $V$ is a faithful, irreducible representation of dimension $n$, is $O\left(n^{\epsilon}\right)$.

If $G$ is either a connected algebraic group or a compact Lie group, let $\mathcal{M}(G)$ denote the set of conjugacy classes of maximal closed subgroups of $G$ (i.e. maximal subgroups among closed proper subgroups). The previous result can be used to give bounds for $|\mathcal{M}(G)|$ in characteristic 0 . Considering parabolic subgroups of algebraic groups shows that $|\mathcal{M}(G)|$ is greater than the rank of $G$. Using the previous result, we can prove:

Theorem 5. Let $G$ be a simple algebraic group over an algebraically closed field of characteristic 0 or a compact Lie group of rank $r$. Then $|\mathcal{M}(G)|=O(r)$.

With some effort, one could get a more precise upper bound in the previous result. The previous result uses a result of Häsä $[\underline{H}]$ which does depend upon the classification of finite simple groups. The result for positive dimensional maximal closed subgroups does not depend on this. See [GLT] for a weaker version of the previous theorem in positive characteristic.

The last result of the paper is about dimensions of fixed spaces of elements in compact groups. The first author and Malle [GM] showed that if $G$ is an irreducible subgroup of $\operatorname{GL}(n, k), n>1$ (for any field $k$ ), then there is an element $g \in G$ with fixed space of dimension at most $n / 3$ (the example $\mathrm{SO}(3)$ shows that this is best possible). This answered a conjecture of Peter Neumann. We show that as $n$ increases we can do much better for Lie groups. Let $w(g)=w_{V}(g)$ denote the largest dimension of any eigenspace of $g$ acting on $V$.

Theorem 6. Let $\epsilon>0$. There exists a positive integer $N=N(\epsilon)$ such that if $G$ is a simple compact Lie group and $V$ is a finite dimensional irreducible $G$ module with $\operatorname{dim} V>N$, then for a dense open subset $X$ of $G, g \in X$ implies that $w_{V}(g)<\epsilon \operatorname{dim} V$.

This immediately implies that the same result holds for algebraic groups over algebraically closed fields of characteristic 0 .

\section{Counting Representations}

We begin by noting that if $G$ is a simple compact Lie group, every representation of $G$ can be regarded as a representation of its universal covering group $\tilde{G}$. Thus,

$$
R_{n}(\tilde{G}) \geq R_{n}(G)
$$

for all $n$, and

$$
\zeta_{\tilde{G}}(s) \geq \zeta_{G}(s)
$$

for all $s>0$ for which the left hand side is defined. Consequently, it suffices to prove the first two theorems for simply connected simple compact Lie groups, and we will assume henceforth that $G=\tilde{G}$. This is immediate for Theorem 22, for Theorem 11, 
we note that if $G$ is a non-trivial quotient of $\mathrm{SU}(2)$ (resp. $\mathrm{SU}(3)$ ), $R_{n}(G)<n$ for $n \geq 2$ (resp. for $n=3$ ). We therefore consider only simply connected groups henceforth.

The irreducible characters $\chi$ of $G$ are indexed by dominant weights of $G$. Let $r$ be the rank of $G$, and $\varpi_{1}, \ldots, \varpi_{r}$ the fundamental weights. Then the dominant weights are the non-negative linear combinations

$$
\lambda=\left(c_{1}-1\right) \varpi_{1}+\cdots+\left(c_{r}-1\right) \varpi_{r},
$$

where the $c_{i}$ are positive integers. The half sum $\rho$ of positive roots equals the sum of the fundamental weights [B, VI, §1, Prop. 29], so

$$
c_{1} \varpi_{1}+\cdots+c_{r} \varpi_{r}=\lambda+\rho .
$$

Weyl's dimension formula for the character $\chi_{\lambda}$ of highest weight $\lambda$ asserts that

$$
\chi_{\lambda}(1)=\prod_{\alpha \in \Phi^{+}} \frac{\alpha^{\vee}(\lambda+\rho)}{\alpha^{\vee}(\rho)},
$$

where $\Phi^{+}$denotes the set of positive roots of $G$, and $\alpha^{\vee}$ denotes the dual root of $\alpha$. We can express this in terms of the $c_{i}$ as follows. If $\alpha_{1}^{\vee}, \ldots, \alpha_{r}^{\vee}$ are the simple positive roots of the dual root system $\Phi^{\vee}$, and $\phi_{1}^{\vee}, \ldots, \phi_{u}^{\vee}$ are all the positive roots in $\Phi^{\vee}$, then every root $\beta_{i}^{\vee} \in \Phi^{\vee}$ can be written uniquely as

$$
\beta_{i}^{\vee}=\sum_{j=1}^{r} a_{i, j} \alpha_{j}^{\vee}
$$

where the $a_{i, j}$ are non-negative integers. As $\alpha_{i}^{\vee}\left(\varpi_{j}\right)=\delta_{i j}$ [B, VI, §1.10], we have

$$
\chi_{\lambda}(1)=\prod_{i=1}^{u} \frac{\sum_{j=1}^{r} a_{i, j} c_{j}}{\sum_{j=1}^{r} a_{i, j}}=\prod_{i=1}^{u} \sum_{j=1}^{r} w_{i, j} c_{j},
$$

where the

$$
w_{i, j}=\frac{a_{i, j}}{\sum_{k=1}^{r} a_{i, k}}
$$

are non-negative and sum to 1 .

By the weighted arithmetic-geometric mean inequality,

$$
\sum_{j=1}^{r} w_{i, j} c_{j} \geq \prod_{j=1}^{r} c_{j}^{w_{i, j}}
$$

Multiplying over $i=1,2, \ldots, u$, we obtain

$$
\chi_{\lambda}(1) \geq \prod_{j=1}^{r} c_{j}^{v_{j}}
$$

where

$$
v_{j}=v_{j}\left(\Phi^{\vee}\right)=\sum_{i=1}^{u} w_{i, j} .
$$

Defining

$$
Z_{\Phi}(s)=\prod_{i=1}^{r} \zeta\left(v_{j}(\Phi) s\right)
$$


where $\zeta(s)$ denotes the Riemann zeta-function, we obtain

$$
\begin{aligned}
\zeta_{G}(s) & =\sum_{c_{1}=1}^{\infty} \cdots \sum_{c_{r}=1}^{\infty} \chi_{\left(c_{1}-1\right) \varpi_{1}+\cdots+\left(c_{r}-1\right) \varpi_{r}}(1)^{-s} \\
& \leq \sum_{c_{1}=1}^{\infty} \cdots \sum_{c_{r}=1}^{\infty} c_{1}^{-v_{1} s} \cdots c_{r}^{-v_{r} s}=Z_{\Phi \vee}(s)
\end{aligned}
$$

for all $s$ for which the right hand side converges.

Our next task is to estimate values of $Z_{\Phi}(s)$ for the various root systems $\Phi$.

Lemma 7. If $\Phi$ is of type $A_{r}$ and the simple roots $\alpha_{i}$ are numbered as usual, then

$$
v_{j}=\sum_{i=1}^{j} \sum_{k=j}^{r} \frac{1}{1+k-i} .
$$

Proof. The roots of $\Phi$ are precisely the sums of consecutive simple roots,

$$
\alpha_{i}+\alpha_{i+1}+\cdots+\alpha_{k} .
$$

Each such root contributes $\frac{1}{1+k-i}$ to the sum (2) if $i \leq j \leq k$ and 0 otherwise.

Lemma 8. If $\Phi$ is of type $A$ and $j \leq \frac{r+1}{2}$, then

$$
v_{j}=v_{r+1-j} \geq j \max (1, \log r-\log j) .
$$

Proof. The equality $v_{j}=v_{r+1-j}$ is clear from Lemma 7. If $1 \leq m \leq n \leq j$, then $1 \leq 1+n-m \leq j \leq j+n-m \leq r$. Setting $i=1+n-m$ and $k=j+n-m$, we obtain

$$
v_{j} \geq \sum_{i=1}^{j} \sum_{k=j}^{r} \frac{1}{1+k-i} \geq \sum_{n=1}^{j} \sum_{m=1}^{n} \frac{1}{n}=j .
$$

On the other hand,

$$
v_{j} \geq \sum_{i=1}^{j} \sum_{k=j}^{r} \frac{1}{1+k-i} \geq j \sum_{k=j}^{r} \frac{1}{k} \geq j \int_{j}^{r} \frac{d x}{x} .
$$

Lemma 9. For $s \geq 3$,

$$
\log \zeta(s) \leq 2^{1-s}
$$

Proof. As $x^{-s}$ is convex for $x>0$,

$$
\zeta(s)<1^{-s}+2^{-s}+\int_{5 / 2}^{\infty} x^{-s} d x=1+2^{-s}+\frac{(5 / 2)^{1-s}}{s-1} .
$$

As $s \geq 3$,

so

$$
(5 / 2)(5 / 2)^{-s} \leq(5 / 2)(5 / 4)^{-3} 2^{-s}<\frac{32}{25} \cdot 2^{-s},
$$

$$
\zeta(s)<1+2^{-s}+\frac{2^{1-s}}{2}=1+2 \cdot 2^{-s},
$$

which implies the lemma.

Proposition 10. If $\Phi$ is of type $A_{r}$ with $r \geq 9$, of type $D_{r}$ with $r \geq 5$, or of type $E_{r}$, then

$$
\zeta_{G}(1)<13 / 7 \text {. }
$$


Proof. Table 1 gives a direct machine computation of the values of $Z_{\Phi}(1)=Z_{\Phi \vee}(1)$ to four decimal places.

We begin with type $A_{r}$. Table 1 shows that this inequality is true for $9 \leq r \leq 20$. We therefore assume $r \geq 21$. By (3), Lemma 8, and Lemma 9 ,

$$
\begin{aligned}
\zeta_{G}(1) & \leq \prod_{i=1}^{6} \zeta\left(v_{i}\right)^{2} \prod_{i=7}^{(r+1) / 2} \zeta\left(v_{i}\right)^{2} \leq \prod_{i=1}^{6} \zeta(i \log (21 / i))^{2} \prod_{i=7}^{(r+1) / 2} \zeta\left(v_{i}\right)^{2} \\
& \leq \prod_{i=1}^{6} \zeta(i \log (21 / i))^{2} \prod_{i=7}^{\infty} e^{2^{2-i}}=\prod_{i=1}^{6} \zeta(i \log (21 / i))^{2} e^{1 / 16}<13 / 7 .
\end{aligned}
$$

Next, we consider type $D_{r}$, using the standard numbering of simple roots $\mathrm{B}$, Planche IV]. If $1 \leq i \leq r-2$ and $1 \leq j \leq r$, there is a unique path from the $i$ th to the $j$ th vertex in the Dynkin diagram; its length is $|j-i|$ unless $j=r$, in which case it is $j-1-i=|j-i|-1$. By [B, VI, $\S 1$, Prop. 19, Cor. 3], the sum of the simple roots corresponding to the vertices in this path is again a positive root. It follows that for $1 \leq i \leq r-2$,

$$
v_{i}\left(D_{r}\right) \geq v_{i}\left(A_{r}\right)
$$

If $1 \leq p<q \leq r-1$, then

$$
\sum_{i=p}^{r} \alpha_{i}+\sum_{j=q}^{r-2} \alpha_{j}
$$

is a positive root, and so is

$$
\beta+\sum_{i=p}^{r-2} \alpha_{i},
$$

for $\beta \in\left\{\alpha_{r-1}, \alpha_{r}\right\}$ and $1 \leq p \leq r-1$. For $1 \leq i \leq r-1$, we count positive roots which are sums of $i$ distinct positive roots, including $\alpha_{r}$. There are $\lceil i / 2\rceil-1$ of type (5), with $p=r-(i-k)<r-k=q$ for $1 \leq k<i / 2$, and there is one of type (6), with $p=r-i$. Thus,

$$
v_{r-1}\left(D_{r}\right)=v_{r}\left(D_{r}\right) \geq \sum_{i=1}^{r-1} \frac{\lceil i / 2\rceil}{i} \geq \frac{r-1}{2} .
$$

If $r \geq 11$, then

$$
v_{r-1}\left(D_{r}\right)>v_{r-1}\left(A_{r}\right)>v_{r}\left(A_{r}\right) .
$$

Therefore, in this range, the proposition is implied by the result for type $A_{r}$. For $5 \leq r \leq 10$, it follows from Table 1 .

Likewise, for $E_{6}, E_{7}$, and $E_{8}$, it follows from Table 1.

We can now prove Theorem 1 .

Proof. If $\Phi$ is among the root systems covered by Proposition 10, then the same is true for its dual, and it follows that $\zeta_{G}(1)<13 / 7$. If, for some $n \geq 7$, we have $R_{n}(G) \geq n$, then

$$
\zeta_{G}(1)>1+(n-1) \cdot n^{-1} \geq 13 / 7 .
$$

Thus, $n \leq 6$. However, $\operatorname{dim} G \geq 45>6^{2}$ for all the cases covered by Proposition 10, so $G$ has no non-trivial representations of degree $\leq 6$. 
TABLE $1 . Z_{\Phi}(1)$

\begin{tabular}{|r|r|r|r|}
\hline$A_{9}$ & 1.8558 & $D_{5}$ & 1.7269 \\
$A_{10}$ & 1.7336 & $D_{6}$ & 1.4609 \\
$A_{11}$ & 1.6400 & $D_{7}$ & 1.3244 \\
$A_{12}$ & 1.5667 & $D_{8}$ & 1.2462 \\
$A_{13}$ & 1.5083 & $D_{9}$ & 1.1978 \\
$A_{14}$ & 1.4610 & $D_{10}$ & 1.1160 \\
$A_{15}$ & 1.4221 & $E_{6}$ & 1.2522 \\
$A_{16}$ & 1.3896 & $E_{7}$ & 1.0836 \\
$A_{17}$ & 1.3621 & $E_{8}$ & 1.0178 \\
$A_{18}$ & 1.3386 & & \\
$A_{19}$ & 1.3182 & & \\
$A_{20}$ & 1.3004 & & \\
\hline
\end{tabular}

TABLE 2. $Z_{\Phi}(3 / 4)^{4}$

\begin{tabular}{|l|l|}
\hline$A_{2}$ & 29547000 \\
$A_{3}$ & 194100 \\
$A_{4}$ & 27475 \\
$A_{5}$ & 7055.8 \\
$A_{6}$ & 2414.5 \\
$A_{7}$ & 1001.9 \\
$A_{8}$ & 481.56 \\
$D_{4}$ & 994.94 \\
\hline
\end{tabular}

Again, if $R_{n}(G) \geq n$, we have $\zeta_{G}(3 / 4) \geq n^{1 / 4}$, and therefore

$$
n \leq \zeta_{G}(3 / 4)^{4} \leq Z_{\Phi \vee}(3 / 4)^{4} .
$$

For each simply laced root system of rank $\geq 2$ not covered by Proposition 10, Table 2 indicates the value of $Z_{\Phi}(3 / 4)^{4}=Z_{\Phi \vee}(3 / 4)^{4}$. An exhaustive machine search for representations of degree less than or equal to $Z_{\Phi}(3 / 4)^{4}$ yields precisely one nontrivial case where $R_{n}(G) \geq n$, namely the equality $R_{3}(\mathrm{SU}(3))=3$. Theorem 1 follows for all $G$ with simply laced root systems.

The remaining irreducible root systems all have the same underlying graph as $A_{r}$, and we use the same numbering convention for the simple roots. By [B, VI, $\S 1$, Prop. 19, Cor. 3], $\alpha_{i}+\alpha_{i+1}+\cdots+\alpha_{j}$ is a positive root for all $i \leq j$. Therefore, the dimension of the irreducible representation of $G$ associated to the $r$-tuple $\left(c_{1}, \ldots, c_{r}\right)$ is at least as great as the dimension of the irreducible representation of $\mathrm{SU}(r+1)$ associated to the same $r$-tuple. This proves $R_{n}(G) \leq n$ for root systems of type B, C, F, and G as well. Moreover, in the case $r=2$, the pairs $(1,2)$ and $(2,1)$ give representations of $B_{2}$ and $G_{2}$ of dimension strictly greater than 3 . Thus, the non-simply laced root systems give no additional cases for which $R_{n}(G)=n>1$.

Now we prove Theorem 2 . 
Proof. Since we are taking the limit as $n \rightarrow \infty$, we can exclude the exceptional groups and groups of type $D_{r}, r \leq 10$. Then, if $G$ is of rank $r$ and $s>2 /(r+1)$,

$$
1 \leq \zeta_{G}(s) \leq Z_{\Phi \vee}(s) \leq Z_{A_{r}}(s) .
$$

Thus, it suffices to prove that for all $s>0$,

$$
\lim _{r \rightarrow \infty} Z_{A_{r}}(s)=1 \text {. }
$$

By (4),

$$
Z_{A_{r}}(s)<\prod_{i=1}^{(r+1) / 2} \zeta\left(s \sum_{i=1}^{j} \sum_{k=j}^{r} \frac{1}{1+k-i}\right)^{2}<\prod_{j=1}^{\infty} \zeta(s \max (j, j \log r / j))^{2} .
$$

If we assume $r>(3 / s) e^{3 / s}$, for each positive integer $j$, either $j>3 / s$ or $r / j>e^{3 / s}$, so by Lemma 9 .

$$
Z_{A_{r}}(s)<\exp \left(4 \sum_{j=1}^{\infty} 2^{-s \max (j, j \log r / j)}\right) .
$$

If $N>\exp (1 / s)$ and $r>N^{2}$,

$$
\sum_{j=1}^{\infty} 2^{-s \max (j, j \log r / j)} \leq \sum_{j=1}^{N} 2^{-s j \log N}+\sum_{j=N+1}^{\infty} 2^{-s j} \leq 2^{1-s \log N}+\frac{2^{-s N}}{1-2^{-s}} .
$$

If $s>0$ is fixed and $N \rightarrow \infty$, the right hand side goes to 0 . This implies (7).

Lemma 11. Let $\epsilon>0$, and let $a_{1}, a_{2}, \ldots$ be a sequence of non-negative real numbers whose growth is $O\left(n^{\epsilon}\right)$. Then

$$
b_{n}:=\sum_{\substack{d_{1} \cdots d_{k}=n \\ 1<d_{1} \leq \cdots \leq d_{k}}} a_{d_{1}} \cdots a_{d_{k}}
$$

also has growth $O\left(n^{\epsilon}\right)$.

Proof. If

$$
f(s):=1^{-s}+\sum_{n=2}^{\infty} a_{n} n^{-s}
$$

converges for all $s>0$, then it converges absolutely for $s>0$, so

$$
\prod_{n=2}^{\infty} \frac{1}{1-a_{n} n^{-s}}=1+\sum_{n=2}^{\infty} b_{n} n^{-s}
$$

converges for all $s>0$. If $a_{n}=O\left(n^{\epsilon}\right)$ for all $\epsilon>0$, then $f(s)$ converges for all $s>0$, so $b_{n}=O\left(n^{\epsilon}\right)$ for all $\epsilon>0$.

Lemma 12. For all positive integers $n$ there exist integers $R$ and $m$ and a finite set $\Lambda \subset \mathbb{N}^{m}$ such that if $G$ is a simply connected, simple, compact Lie group of rank $r \geq R$ and $\chi_{\lambda}(1) \leq r^{n}$, then either $G$ is of type $A_{r}$ and

$$
\lambda=a_{1} \varpi_{1}+\cdots+a_{m} \varpi_{m}+b_{m} \varpi_{r+1-m}+\cdots+b_{1} \varpi_{r}
$$

for some $\left(a_{1}, \ldots, a_{m}\right),\left(b_{1}, \ldots, b_{m}\right) \in \Lambda$, or $G$ is of type $B_{r}, C_{r}$, or $D_{r}$, and

$$
\lambda=a_{1} \varpi_{1}+\cdots+a_{m} \varpi_{m}
$$

for some $\left(a_{1}, \ldots, a_{m}\right) \in \Lambda$. 
Proof. For $1 \leq i \leq r$,

$$
v_{i}(\Phi) \geq 1+1 / 2+\cdots+1 / r-1>\log r .
$$

By (11), this implies that $c_{i} \leq m / \log 2$ for all $i$. Our goal is therefore to prove that $c_{i}=1$ except when $i$ is bounded above or, in the case of $A_{r}$, when $r-i$ is bounded above. For $A_{r}$, this is an easy consequence of Lemma 7 . For the remaining root systems, we need only prove that for every constant $C, v_{i}(\Phi) \leq C \log r$ implies an upper bound on $i$. For $B_{r}$, by [B, Planche II], for $1 \leq p<q \leq r$,

$$
\sum_{i=p}^{q-1} \alpha_{i}+2 \sum_{j=q}^{r} \alpha_{i} \in \Phi^{+} .
$$

Thus, the sum for $v_{i}\left(B_{r}\right)$ contains at least $i(2 r-1-i) / 2$ terms, each of which is at least $1 /(2 r-1)$. Thus,

$$
v_{i}\left(B_{r}\right) \geq \max \left(v_{i}\left(A_{r}\right), \frac{i(r-1)}{2 r-1}\right),
$$

so $v_{i}\left(B_{r}\right) \leq C \log r$ implies an upper bound on $i$ independent of $r$. For $C_{r}$, by [B, Planche III], for $1 \leq p<q \leq r$,

$$
\alpha_{r}+\sum_{i=p}^{q-1} \alpha_{i}+\sum_{j=q}^{r-1} \alpha_{i} \in \Phi^{+} .
$$

As in the $B_{r}$ case, $v_{i}\left(C_{r}\right)$ is the sum of at least $i(2 r-1-i) / 2$ terms, each of which is at least $1 /(2 r-1)$, so

$$
v_{i}\left(C_{r}\right) \geq \max \left(v_{i}\left(A_{r}\right), \frac{i(r-1)}{2 r-1}\right),
$$

which implies an upper bound on $i$, independent of $r$. By (5),

$$
v_{i}\left(D_{r}\right) \geq \max \left(v_{i}\left(A_{r}\right), \frac{i(r-1)}{2 r-3}\right),
$$

for $1 \leq i \leq r-2$, and

$$
v_{r-1}\left(D_{r}\right)=v_{r}\left(D_{r}\right) \geq \frac{(r-1)(r-2)}{2 r-3},
$$

which again implies an upper bound on $i$, independent of $r$.

We now prove Theorem 4 .

Proof. If $G$ is a semisimple compact Lie group with universal covering group $\tilde{G}$ and $V$ is a faithful irreducible representation of $G$, then we can regard $V$ as an almost faithful irreducible representation of $\tilde{G}$. Writing $\tilde{G}$ as a product of its simple factors $G_{1} \times \cdots \times G_{k}$, we can factor $V$ as $V_{1} \otimes \cdots \otimes V_{k}$, where each $V_{i}$ is a non-trivial representation. We can order the factors $G_{i}$ so that $\operatorname{dim} V_{1} \leq \operatorname{dim} V_{2} \leq$ $\cdots \leq \operatorname{dim} V_{k}$. By Lemma 11, it suffices to prove that for each $n$, the number of pairs $(G, V)$ where $G$ is a simply connected, simple, compact Lie group and $V$ is a representation of dimension $n$ is $O\left(n^{\epsilon}\right)$ for all $\epsilon>0$.

By Theorem 2, for every $\epsilon>0$, there exists a finite set $\Sigma$ of groups such that for every simply connected, simple, compact Lie group $G$ not isomorphic to any element of $\Sigma$, we have $\zeta_{G}(\epsilon / 2) \leq 2$. For any such $G, R_{n}(G) \leq 2 n^{\epsilon / 2}$. If we limit ourselves to groups $G$ of rank $\leq n^{\epsilon / 2}$ which do not lie in $\Sigma$, the total contribution is bounded by $2 n^{\epsilon}$. 
Next we show that for each $G \in \Sigma$ there are $O\left(n^{\epsilon}\right)$ representations of $G$ of degree $n$. Suppose that $G$ has rank $r$. The representations of $G$ are indexed by $\left(c_{1}, \ldots, c_{r}\right) \in \mathbb{N}^{r}$. It suffices to show that for each $j \in\{1, \ldots, r\}$, the number of representations with $c_{j}>0$ and degree $n$ is $O\left(n^{\epsilon}\right)$. Applying [B] VI, 1 , Prop. 19, Cor. 3], for each $k \in\{1, \ldots, r\}$, the sum of all the simple roots corresponding to vertices on the path from $j$ to $k$ is a positive root $\beta_{k}$. Clearly, $\beta_{1}, \ldots, \beta_{r}$ span the root system. Ordering the positive roots of $G$ so that $\beta_{1}, \ldots, \beta_{r}$ come first, we deduce from Weyl's dimension formula that

$$
\prod_{i=1}^{r} \sum_{j=1}^{r} a_{i, j} c_{j} \mid C_{G} n
$$

where

$$
C_{G}=\prod_{i=1}^{u} \sum_{j=1}^{r} a_{i, j} .
$$

Moreover, the linear independence of $\beta_{1}, \ldots, \beta_{r}$ implies that the $r$-tuple

$$
\left(d_{1}, \ldots, d_{r}\right)=\left(\sum_{j=1}^{r} a_{1, j} c_{j}, \ldots, \sum_{j=1}^{r} a_{r, j} c_{j}\right)
$$

determines $\left(c_{1}, \ldots, c_{r}\right)$. We define

$$
d_{r+1}=\frac{C_{G} n}{d_{1} \cdots d_{n}}
$$

and then let $e_{1}, \ldots, e_{s}, s \leq r+1$, denote the sequence obtained by ordering all elements in the set $\left\{d_{1}, \ldots, d_{r+1}\right\}$ which are greater than 1 . Thus

$$
\sum_{d_{1} \cdots d_{r} \mid C_{G} n} 1 \leq(r+1) ! \sum_{\substack{e_{1} \cdots e_{s}=C_{G} n \\ 1<e_{1} \leq e_{2} \leq \cdots \leq e_{s}}} 1 .
$$

By Lemma 11, this sum is $O\left(\left(C_{G} n\right)^{\epsilon}\right)$ and therefore $O\left(n^{\epsilon}\right)$.

This leaves only groups of rank $\geq n^{\epsilon / 2}$, and taking $n$ sufficiently large, we may assume that only classical groups need be considered. Setting $m=2 / \epsilon$ in Lemma 12 , there exists a finite set $\Lambda$, so that if $G$ is of type $A_{r}, \lambda$ is given by (8), and if $G$ is of type $B_{r}, C_{r}$, or $D_{r}$, it is given by (9). We claim that for each element $\left(a_{1}, \ldots, a_{m}\right) \in \Lambda$ and each family $\mathrm{B}, \mathrm{C}, \mathrm{D}$, the dimension of the representation is a strictly monotone function of $r$; in case $\mathrm{A}$, the analogous statement is true for each pair $\left(a_{1}, \ldots, a_{m}\right),\left(b_{1}, \ldots, b_{m}\right) \in \Lambda$. Perhaps the easiest way to see this is to note that the branching rules for classical groups [ $\mathrm{K}$, 9.14, 9.16, 9.18] imply that for $X \in\{A, B, C, D\}$, the restriction of the representation of $X_{r}$ associated to $\left(a_{1}, \ldots, a_{m}\right)$ to $X_{r-1}$ contains the representation of $X_{r-1}$ associated to $\left(a_{1}, \ldots, a_{m}\right)$ as a proper subrepresentation. We conclude that the groups of rank $\geq n^{\epsilon / 2}$ contribute at most a constant term $|\Lambda|^{2}+3|\Lambda|$ to the total number of pairs $(G, V)$.

\section{Maximal Subgroups of Classical groups}

Let $k$ be an algebraically closed field of characteristic $p \geq 0$. Let $G$ be a simple classical group over $k$ (i.e. $G=\mathrm{SL}(n), n>1, \operatorname{Sp}(2 n), n>1, \mathrm{SO}(2 n), n>3$, $\mathrm{SO}(2 n+1), n>2, p \neq 2)$. Table 3 gives Aschbacher's description of proper subgroups in the case of algebraic groups. See [A, KL, G, GT, LSe. Let $V$ denote the natural module for $G$. If $H$ is a proper closed subgroup of $G$, then $H$ belongs to 
TABle 3. Aschbacher Classes

\begin{tabular}{|l|l|}
\hline $\mathcal{C}_{1}$ & $H$ preserves a totally singular or non-degenerate subspace of $V$ \\
\hline $\mathcal{C}_{2}$ & $H$ preserves an additive decomposition of $V$ \\
\hline $\mathcal{C}_{4}$ & $V$ is tensor decomposable for $H$ \\
\hline $\mathcal{C}_{6}$ & $H$ normalizes a subgroup of symplectic type \\
\hline $\mathcal{C}_{7}$ & $V$ is tensor induced for $H$ \\
\hline $\mathcal{C}_{8}$ & $V$ is the natural module for a classical subgroup $H$ \\
\hline $\mathcal{S}_{1}$ & $H$ is the normalizer of a quasisimple finite group $S$ \\
\hline $\mathcal{S}_{2}$ & $H$ is the normalizer of a simple algebraic subgroup $S$ \\
\hline
\end{tabular}

one of the classes $\mathcal{C}_{i}(G)$ or $\mathcal{S}_{i}(G)$ (note that for the last two classes, we may also assume that $S$ acts irreducibly or we would be in one of the other cases).

Note that we have kept the same numbering as in Aschbacher's theorem for finite groups but certain cases do not arise in the algebraic group case.

It is easy to see, as in the proof [LPS, Lemmas 2.1, 2.4], that:

Lemma 13. Let $G$ be a classical group over $k$ with $n$ being the dimension of the natural module $V$. The number of conjugacy classes of maximal subgroups of $G$ in $\bigcup_{H \in \mathcal{C}_{i}}$ is at most $n+3 \log n+3$.

We need to deal with the maximal subgroups in $\mathcal{S}_{1}$ and $\mathcal{S}_{2}$. Unfortunately in positive characteristic, one has much less information available about dimensions of irreducible representations. See [GLT] for a weaker version of these results. Now we assume that we are in characteristic 0 .

By Theorem 4, it follows that the number of conjugacy classes of maximal subgroups in $\mathcal{S}_{2}(G)$ is $O\left(n^{\epsilon}\right)$.

The result for $\mathcal{S}_{1}$ we need has recently been obtained by Häsä [ $\left.\mathrm{H}\right]$. This is the only result in the paper that requires the classification of finite simple groups.

Lemma 14. The number of conjugacy classes of maximal subgroups in $\mathcal{S}_{1}(G)$ is at most $O(n)$.

Now Theorem 5 for algebraic groups follows, for if the rank of $G$ is fixed, there is nothing to prove. So we may assume that $G$ is a classical group in sufficiently large dimension, and the remarks above immediately give the desired bound.

It is now quite easy to obtain the result for compact groups. A nice observation of Nolan Wallach shows that the Cartan decomposition for complex algebraic groups [W1, Lemma 2.1.5] gives:

Lemma 15. Let $G$ be a compact real Lie group and $G_{\mathbb{C}}$ its complexification. If $A, B$ are subgroups of $G$ and are conjugate in $G_{\mathbb{C}}$, then they are conjugate in $G$.

It follows that the number of conjugacy classes of maximal closed subgroups of a compact real Lie group is the number of conjugacy classes of maximal closed reductive subgroups of $G_{\mathbb{C}}$. Assume that $G$ (and so also $G_{\mathbb{C}}$ ) is connected. Suppose that $M$ is a maximal reductive subgroup of $G_{\mathbb{C}}$ but is not maximal. Then $M<X$, where $X$ has a unipotent radical. By the Borel-Tits lemma, this implies that $M<P$, where $P$ is a parabolic subgroup with unipotent radical $Q$. Since $M$ is reductive, $M \cap Q=1$. Let $L$ be a Levi subgroup of $Q$. Set $H=M Q \cap L$. Then $H Q=M Q$. Since $H^{1}(M, V)=0$ for any $H$-invariant section of $A$, it follows that $H$ and $M$ are conjugate. Thus, by maximality, $M$ is conjugate to $L$. Since $M$ 
is maximal reductive, it follows that $P$ is a maximal parabolic. This shows that the number of conjugacy classes of maximal closed subgroups of $G$ is at most the number of conjugacy classes of maximal closed subgroups of $G_{\mathbb{C}}$, whence the result. Note that it is possible that $G$ has strictly fewer conjugacy classes of maximal closed subgroups than $G_{\mathbb{C}}$, since Levi subgroups need not be maximal reductive subgroups and the Levi subgroups of non-conjugate parabolic subgroups may be conjugate. See [GW] for more details.

\section{Dimensions of FIXED SPACES}

In this section, we prove Theorem 6 . Let $G$ be a simple compact Lie group and $V$ a non-trivial irreducible finite dimensional module for $G$. The minimum value of $w_{V}(g)$ as $g$ ranges over $G$ is the maximal dimension of the weight spaces of $V$, and this value is achieved on a dense open subvariety of $G$. Therefore, it suffices to exhibit just one $g$ satisfying the asserted inequality.

We require two lemmas. The first is due to Seitz $[\underline{S}$ (and the result holds in all characteristics and for finite Chevalley groups as well).

Lemma 16. Let $T$ be a maximal torus of $G$. Then the dimension of any weight space for $T$ is at most $1+(\operatorname{dim} V) / h$, where $h$ is the Coxeter number of $G$.

This proves the theorem if the rank is increasing. So it suffices to prove the theorem for a fixed $G$. Daniel Goldstein Go proved Theorem [6 in this case. We give a different proof (Goldstein's proof gives somewhat better bounds).

Lemma 17. Let $G$ be a simple compact Lie group. Let $V$ be an irreducible finite dimensional module for $G$. Let $g \in G$ be a regular element of prime order $p$. Then

$$
\limsup _{\operatorname{dim} V \rightarrow \infty} \frac{w_{V}(g)}{\operatorname{dim} V}=\frac{1}{p} .
$$

Proof. Let $\chi$ denote the character of $V$. By the Weyl character formula,

$$
\left|\chi\left(g^{k}\right)\right| \leq \frac{|W|}{\left.\left|1-e^{2 \pi i / p}\right|\right|^{+\Phi^{+}}}
$$

for $1 \leq k \leq p-1$, where $\Phi^{+}$is the set of positive roots of the root system of $G$ and $W$ is the Weyl group. Let $\lambda$ be any irreducible character for $L:=\langle g\rangle$. If $m(\lambda, \chi)$ is the multiplicity of $\lambda$ in the restriction of $\chi$ to $L$, then

$$
p m(\lambda, \chi)=\sum_{k=0}^{p-1} \chi\left(g^{k}\right) \lambda\left(g^{-k}\right) \leq \chi(1)+(p-1) \frac{|W|}{\left|1-e^{2 \pi i / p}\right|^{\left|\Phi^{+}\right|}} .
$$

Note that in its action on $V, g$ has only $p$ eigenvalues, so

$$
w_{V}(g)=\max _{\lambda}\{m(\lambda, \chi)\} \geq \chi(1) / p .
$$

Combining this with (10), the result follows.

Since for any sufficiently large prime $p$ there are regular elements of order $p$ in $G$, we see that there is some element $g \in G$ with $w(g)<\epsilon \operatorname{dim} V$ as long as $\operatorname{dim} V$ is sufficiently large. This completes the proof. 


\section{REFERENCES}

[A] Aschbacher, Michael: On the maximal subgroups of the finite classical groups, Invent. Math. 76 (1984), 469-514. MR746539 (86a:20054)

[B] Bourbaki, N.: Éléments de mathématique. Fasc. XXXIV. Groupes et algèbres de Lie. Chapitre IV: Groupes de Coxeter et systèmes de Tits. Chapitre V: Groupes engendrés par des réflexions. Chapitre VI: systèmes de racines. Actualités Scientifiques et Industrielles, No. 1337, Hermann, Paris, 1968. MR0240238 (39:1590)

[Go] Goldstein, Daniel: personal communication.

[G] Guralnick, Robert: Monodromy groups of coverings of curves, Galois groups and fundamental groups, 1-46, Math. Sci. Res. Inst. Publ., 41, Cambridge Univ. Press, Cambridge, 2003. MR.2012212 (2004j:14029)

[GLT] Guralnick, Robert; Larsen, Michael; Tiep, Pham Huu: Representation growth in positive characteristic and conjugacy classes of maximal subgroups, preprint, arXiv:1009.2437.

[GM] Guralnick, Robert; Malle, Gunter: Products of conjugacy classes and fixed point spaces, J. Amer. Math. Soc., to appear, arXiv:1005.3756.

[GT] Guralnick, Robert; Tiep, Pham Huu: Symmetric powers and a problem of Kollár and Larsen, Invent. Math. 174 (2008), 505-554. MR2453600(2009j:20065)

[GW] Guralnick, Robert; Wallach, Nolan: Subgroups of real reductive groups, preprint.

$[\mathrm{H}] \quad$ Häsä, Jokke: Representation growth of finite quasisimple groups, preprint.

[K] Knapp, Anthony W.: Lie groups beyond an introduction. Second edition. Progress in Mathematics, 140. Birkhäuser Boston, Inc., Boston, MA, 2002. MR.1920389 (2003c:22001)

[KL] Kleidman, Peter; Liebeck, Martin: The subgroup structure of the finite classical groups, London Mathematical Society Lecture Note Series, 129. Cambridge University Press, Cambridge, 1990. MR1057341 (91g:20001)

[L] Lübeck, Frank: Small degree representations of finite Chevalley groups in defining characteristic, LMS J. Comput. Math. 4 (2001), 135-169. MR.1901354(2003e:20013)

[LL] Larsen, Michael; Lubotzky, Alexander: Representation Growth for Linear Groups, J. Eur. Math. Soc. 10 (2008), no. 2, 351-390. MR2390327 (2009b:20080)

[LPS] Liebeck, Martin W.; Pyber, Laszlo; Shalev, Aner: On a conjecture of G. E. Wall, J. Algebra 317 (2007), 184-197. MR2360145 (2009b:20048)

[LS] Liebeck, Martin W.; Shalev, Aner: Character degrees and random walks in finite groups of Lie type, Proc. London Math. Soc. (3) 90 (2005), no. 1, 61-86. MR2107038|(2006h:20016)

[LSe] Liebeck, Martin W.; Seitz, Gary M.: On the subgroup structure of classical groups. Invent. Math. 134 (1998), 427-453. MR1650328 (99h:20074)

[S] Seitz, Gary M.: Bounds for dimensions of weight spaces of maximal tori, Linear algebraic groups and their representations (Los Angeles, CA, 1992), 157-161, Contemp. Math., 153, Amer. Math. Soc., Providence, RI, 1993. MR.1247503 (94m:20097)

[W1] Wallach, Nolan: Real reductive groups. I. Pure and Applied Mathematics, 132. Academic Press, Inc., Boston, MA, 1988. MR.929683 (89i:22029)

[W2] Wallach, Nolan: personal communication.

Department of Mathematics, University of Southern California, 3620 South Vermont Avenue, Los Angeles, California 90089-2532

E-mail address: guralnic@usc.edu

Department of Mathematics, Indiana University, Bloomington, Indiana 47405

E-mail address: mjlarsen@indiana.edu

Department of Mathematics, Indiana University, Bloomington, Indiana 47405

Current address: Department of Mathematics, Amherst College, Amherst, Massachusetts 01002-5000

E-mail address: cmanack1@gmail.com 